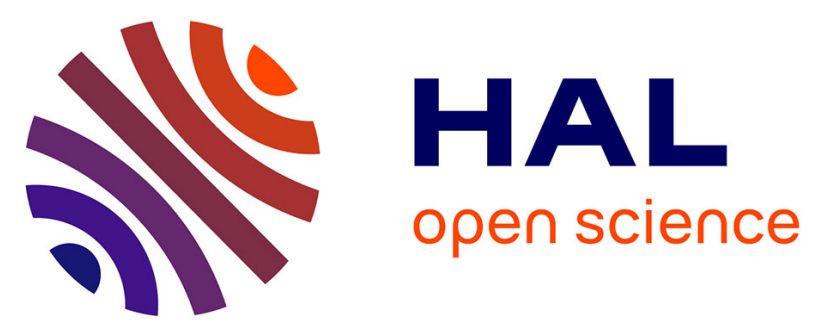

\title{
Joint Assessment of Quantitative 18F-Florbetapir and 18F-FDG Regional Uptake Using Baseline Data from the ADNI
}

Fayçal Ben Bouallègue, Denis Mariano-Goulart, Pierre Payoux

\section{- To cite this version:}

Fayçal Ben Bouallègue, Denis Mariano-Goulart, Pierre Payoux. Joint Assessment of Quantitative 18F-Florbetapir and 18F-FDG Regional Uptake Using Baseline Data from the ADNI. Journal of Alzheimer's Disease, 2018, 62 (1), pp.399 - 408. 10.3233/JAD-170833 . hal-01786614

\section{HAL Id: hal-01786614 \\ https://hal.umontpellier.fr/hal-01786614}

Submitted on 21 Apr 2020

HAL is a multi-disciplinary open access archive for the deposit and dissemination of scientific research documents, whether they are published or not. The documents may come from teaching and research institutions in France or abroad, or from public or private research centers.
L'archive ouverte pluridisciplinaire HAL, est destinée au dépôt et à la diffusion de documents scientifiques de niveau recherche, publiés ou non, émanant des établissements d'enseignement et de recherche français ou étrangers, des laboratoires publics ou privés. 


\title{
Joint Assessment of Quantitative
} ${ }^{18}$ F-Florbetapir and ${ }^{18}$ F-FDG Regional Uptake Using Baseline Data from the ADNI

Fayçal Ben Bouallègue ${ }^{\mathrm{a}, \mathrm{b}, \mathrm{c}, *}$, Denis Mariano-Goulart ${ }^{\mathrm{a}, \mathrm{c}}$, Pierre Payoux $^{\mathrm{b}, \mathrm{d}}$ and for the Alzheimer's Disease Neuroimaging Initiative (ADNI) ${ }^{1}$

${ }^{a}$ Department of Nuclear Medicine, Montpellier University Hospital, Montpellier, France

${ }^{\mathrm{b}}$ Department of Nuclear Medicine, Purpan University Hospital, Toulouse, France

${ }^{\mathrm{c}}$ PhyMedExp, INSERM, CNRS, Montpellier University, Montpellier Cedex, France

${ }^{\mathrm{d}}$ ToNIC, Toulouse NeuroImaging Center, Université de Toulouse, Inserm, UPS, Toulouse, France

\begin{abstract}
Joint analysis of amyloid and metabolic PET patterns across healthy, mild cognitive impairment (MCI), and Alzheimer's disease (AD) subjects was performed using baseline ${ }^{18} \mathrm{~F}$-florbetapir and ${ }^{18} \mathrm{~F}$-FDG PET of 684 subjects from the ADNI (251 normal, 204 stable MCI, 85 AD converters, and 144 AD). Correlation between regional amyloid and metabolic uptake was measured and predictive value of PET profile regarding AD conversion in cognitively impaired subjects was assessed using survival analysis and support vector machine classification (SVM). The highest correlations were found in the temporal cortex, precuneus, and posterior cingulum. With respect to normal controls, amyloid load increase was diffuse and early in MCI subjects, whereas metabolism decrease occurred later and predominated in temporo-parietal, precuneus, and cingulate cortices. Five-year AD conversion rates in cognitively impaired subjects were 5\%,22\%, 42\%, and 78\% in amyloid/FDG-, amyloid-/FDG+, amyloid+/FDG-, and amyloid+/FDG+ subjects respectively (mean follow-up $37 \pm 14$ months). Using SVM, the combination of ADAS-cog score, amyloid PET, and FDG PET yielded better performance in predicting AD conversion (77\% accuracy; $58 \%$ positive predictive value; $88 \%$ negative predictive value) than ADAS-cog $(72 \% ; 52 \% ; 86 \%$ ), amyloid PET $(72 \% ; 52 \% ; 87 \%)$, and FDG PET $(67 \% ; 47 \% ; 84 \%)$. This study attests the complementary value of amyloid and FDG PET in MCI assessment and the efficiency of combined cognitive, amyloid, and metabolic scores to predict AD conversion.
\end{abstract}

Keywords: Alzheimer's disease, Alzheimer's Disease Neuroimaging Initiative, amyloid PET, FDG PET, mild cognitive impairment

\footnotetext{
${ }^{1}$ Data used in preparation of this paper were obtained from the Alzheimer's Disease Neuroimaging Initiative (ADNI) database (http://adni.loni.usc.edu/). As such, the investigators within the ADNI contributed to the design and implementation of ADNI and/or provided data but did not participate in analysis or writing of this paper. A complete listing of ADNI investigators can be found at http://adni.loni.usc.edu/wp-content/ uploads/how_to_apply/ADNI_Acknowledgement_List.pdf.

*Correspondence to: Fayçal Ben Bouallègue, Lapeyronie University Hospital, Nuclear Medicine Department, Avenue du Doyen Giraud, 34295 Montpellier Cedex 5, France. Tel.: +33 467338598; Fax: +33 467338465; E-mail: faybenb@ hotmail.com.
}

\section{INTRODUCTION}

Alzheimer's disease (AD) is characterized by the accumulation of amyloid plaques and neurofibrillary tangles in the brain inducing a progressive neuronal dysfunction and degeneration [1,2]. These slow neuropathological alterations initiate early in the natural history of the disease and determine a long lasting prodromal state of mild cognitive impairment (MCI) $[3,4]$. The study of structural and functional AD-related brain changes has benefited from 
the development of neuroimaging techniques. Accurate and reproducible measures of regional gray matter atrophy are obtained using high-resolution magnetic resonance (MR) imaging [5, 6]. Positron emission tomography (PET) allows to evaluate cortical amyloid load [7, 8] and glucose metabolism as a surrogate of neurodegeneration [9, 10] using amyloid tracers (such as ${ }^{18} \mathrm{~F}$-florbetapir) and ${ }^{18} \mathrm{~F}$ fuorodeoxyglucose (FDG), respectively. Although the debate remains open [11], currently available evidence strongly supports the hypothesis that the primum movens in AD is related to abnormal processing of amyloid- $\beta(A \beta)$ peptide and formation of $A \beta$ plaques during the asymptomatic and prodromal stages of the disease. After a lag period, which varies from patient to patient, neurodegeneration becomes the dominant pathological processes, accompanied by synaptic dysfunction and correlative decreased glucose metabolism $[12,13]$.

Recent multimodal imaging research examining the relations between regional profiles of amyloid deposition and hypometabolism in healthy, MCI, and AD subjects tended to confirm this pathophysiological model [14-24]. Numerous studies, however, focused specifically either on healthy [19], MCI $[14,18,23,24]$, or AD subjects [17]. Most of studies comparing PET measurements of amyloid load and hypometabolism with respect to longitudinal cognitive decline have been limited by small sample sizes [15, 16, 23, 24]. In a large cohort of subjects from the Alzheimer's Disease Neuroimaging Initiative (ADNI), Landau et al. examined the cross-sectional relationships between amyloid deposition, hypometabolism, and cognition, as well as the associations between global amyloid and hypometabolism PET measurements and retrospective cognitive decline [25]. Their results suggested that amyloid deposition has an early and subclinical impact on cognition that precedes metabolic changes, whereas at later stages of the disease hypometabolism becomes more pronounced and more closely linked to cognitive decline.

To date, accurate data regarding the local and regional correlation between amyloid deposition and hypometabolism patterns across subjects at various stages of the disease is lacking, as remains unclear the exact prognostic value of joint amyloid and metabolic profile. We sought to evaluate cortical amyloid load and metabolism in a large cohort of ADNI subjects, including healthy, stable MCI, AD converters, and AD subjects. This was done by assessing the correlation between amyloid and FDG PET measurements and studying the differences in terms of joint amyloid and metabolic profile across cross-sectionally modelled disease stages. The predictive value of quantitative amyloid and FDG PET with regards to AD conversion in cognitively impaired patients was further evaluated.

\section{MATERIALS AND METHODS}

\section{Subjects}

In this study, we used participant data from the ADNI, a multicenter project with approximately 50 medical centers and university sites across the United States and Canada [26]. The ADNI was launched in 2003 as a public-private partnership, led by Principal Investigator Michael W. Weiner, MD. Its primary goal was to examine how brain imaging and other biomarkers can be used to measure the progression of MCI and early AD. A detailed description of the inclusion criteria can be found on the ADNI webpage (http://adni-info.org). Subjects were between 55 and 90 years old and willing and able to undergo all test procedures including neuroimaging. The study was approved by the Institutional Review Boards of all of the participating institutions and informed written consent was obtained from all participants at each site. Cognitively normal participants were the control subjects in the ADNI study. Significant memory complaint (SMC) participants scored within the normal range for cognition but indicated concerns, and exhibited slight forgetfulness. They showed no signs of depression, MCI, or dementia. MCI participants reported a subjective memory concern either autonomously or via an informant or clinician. However, other cognitive domains showed no significant impairment, activities of daily living were essentially preserved, and there were no signs of dementia. AD subjects (either at baseline or during follow-up) met the NINCDS/ADRDA criteria for probable AD [27, 28].

Data were downloaded from the ADNI database (http://adni.loni.usc.edu) and included all subjects from the ADNI-2 with available baseline florbetapir PET, FDG PET, and T1-MRI, matching one of the four following profiles: 1) normal: subjects rated normal both at baseline and during follow-up; 2) stable MCI: subjects rated MCI both at baseline and during follow-up with a minimal follow-up duration of 12 months; 3) AD converters: subjects with baseline SMC or MCI who converted to AD during follow-up (two subjects who converted to non-AD 
Table 1

Characteristics of the study population

\begin{tabular}{lcccccc}
\hline & $\mathrm{N}$ & $\begin{array}{c}\text { Male } \\
\text { gender }\end{array}$ & $\begin{array}{c}\text { Age } \\
(\mathrm{y})\end{array}$ & $\begin{array}{c}\text { ApoE4 } \\
\text { carriers }\end{array}$ & $\begin{array}{c}\text { Follow- } \\
\text { up (mo) }\end{array}$ & $\begin{array}{c}\text { ADAS- } \\
\text { cog13 score }\end{array}$ \\
\hline Normal & 251 & $110(43 \%)$ & $73 \pm 6$ & $75(30 \%)$ & $31 \pm 14$ & $9 \pm 4$ \\
Stable MCI & 204 & $117(57 \%)$ & $72 \pm 7$ & $86(42 \%)$ & $37 \pm 14$ & $14 \pm 6$ \\
AD converters & 85 & $45(53 \%)$ & $73 \pm 7$ & $61(72 \%)$ & $36 \pm 13$ & $22 \pm 7$ \\
AD & 144 & $84(58 \%)$ & $75 \pm 8$ & $95(66 \%)$ & $12 \pm 7$ & $31 \pm 9$ \\
\hline
\end{tabular}

dementia during follow-up were excluded from the analysis); 4) $\mathrm{AD}$ : subjects rated $\mathrm{AD}$ both at baseline and during follow-up. Eight subjects for whom the automatic registration of amyloid and/or FDG PET with T1-MRI failed were excluded. Our population included 684 subjects $(251$ normal, 204 stable MCI, $85 \mathrm{AD}$ converters, and $144 \mathrm{AD}$, the complete listing of subject IDs is available at: http://scinti.edu.umont pellier.fr/files/2017/11/JAD_Subject_IDs.xlsx). Cognitively impaired subjects (groups 2 and 3 ) underwent an average clinical follow-up of $37 \pm 14$ months. Table 1 details the characteristics of the study population including ApoE4 status, and Alzheimer's Disease Assessment Scale - Cognitive subscale (ADAS- $\operatorname{cog} 13)$.

\section{Image acquisition and processing}

Amyloid- $\beta$ deposition and glucose metabolism were visualized using ${ }^{18} \mathrm{~F}$-florbetapir and ${ }^{18} \mathrm{~F}$ FDG PET respectively. Technical details regarding PET acquisition and pre-processing analysis have been described in more detail elsewhere [25, 29] and are available online (http://adni.loni.usc.edu/ methods/pet-analysis/). Concurrent T1-MR was used as a structural template to spatially normalize the PET images and define regions of interest (ROI) for each subject using SPM12 (Wellcome Trust Centre, London, UK). Resulting PET data were sampled on a $135 \times 155 \times 128$ grid with cubic voxels of $1.5 \times 1.5 \times 1.5 \mathrm{~mm}^{3}$. PET image voxels were labelled according to the maximum probability tissue atlas derived from the "MICCAI 2012 grand challenge and workshop on multi-atlas labelling" and provided by Neuromorphometrics, Inc. (http:// neuromorphometrics.com) under academic subscription. Amyloid and metabolic standardized uptake values (SUV) were normalized to mean cerebellar uptake to produce SUV ratios (SUVR) [30, 31]. Mean regional SUVRs were computed in the following cortical ROIs (right and left ROIs were averaged): frontal, temporal, parietal, occipital, insular, precuneus, anterior cingulate, and posterior cingulate cortices.

\section{Statistical analyses}

Correlation between amyloid and metabolic SUVRs at the voxel level and in cortical ROIs was assessed using Pearson's coefficient r. Univariate differences in amyloid and metabolic SUVR between the four clinical profiles were assessed using Student's $t$-test. Bivariate differences in joint distribution of amyloid load and glucose metabolism were assessed using Fisher's F-score derived from the $\mathrm{T}^{2}$ Hotelling statistic. Bonferroni correction was used to adjust $p$-values for multiple testing.

Receiver operating characteristic (ROC) analysis was used to determine optimal regional SUVR thresholds as those maximizing Youden's index (i.e., sensitivity + specificity -1$)$. This was done by analyzing groups 1 and 4 (normal and AD subjects) and considering normal subjects with SUVR below (amyloid) or above (FDG) the threshold as true negatives, and $\mathrm{AD}$ subjects with SUVR above (amyloid) or below (FDG) the threshold as true positives. Fiveyear $\mathrm{AD}$ conversion rate in groups 2 and 3 was then assessed according to amyloid and FDG PET profiles using the actuarial method. Regional SUVRs were compared to the optimal thresholds given by the ROC analysis in five cortical areas in which amyloid and FDG anomalies are typically found in MCI and AD subjects $[8,32]$ : frontal, temporal, and parietal cortices, precuneus, and posterior cingulum. Amyloid PET was considered abnormal if an increased SUVR was observed in at least 3 of these 5 areas. FDG PET was considered abnormal if a decreased SUVR was observed in at least 3 of these 5 areas. Conversion hazard as a function of PET profile was assessed using a Cox proportional hazards ratio model accounting for age, gender, ApoE status, and baseline ADAS-cog.

Prognostic value of ADAS-cog score, quantitative amyloid PET pattern, quantitative FDG PET pattern, and their combination regarding conversion to $\mathrm{AD}$ in cognitively impaired subjects (groups 2 and 3 ) 


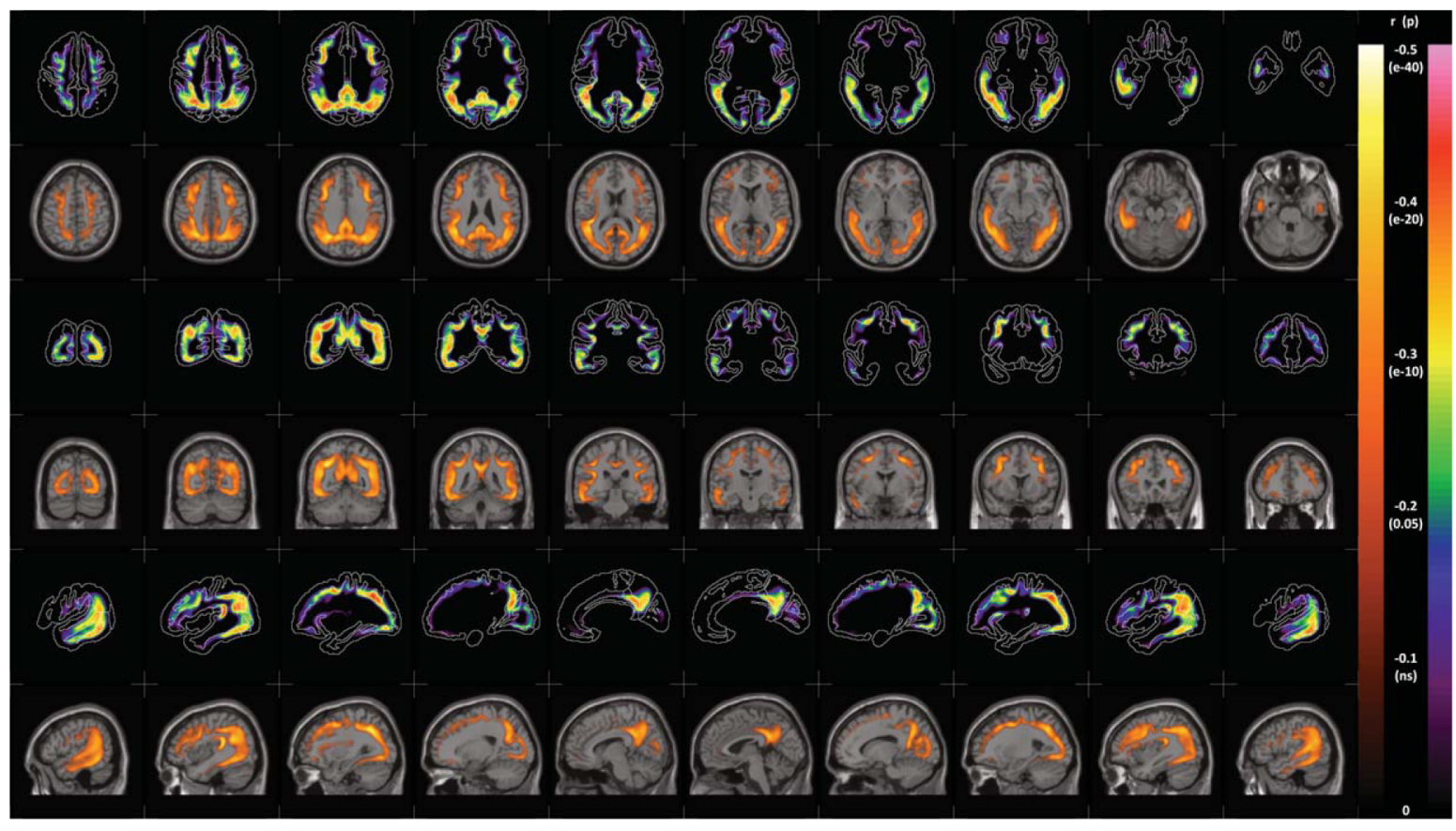

Fig. 1. Voxel-wise correlation (Pearson's r) between amyloid load (florbetapir SUVR) and glucose metabolism (FDG SUVR). Top rows show the correlation map with cortical ROIs superimposed. Bottom rows show the correlation map overlaid on T1-MR slices. $P$-values are corrected for multiple testing (Bonferroni). A color version of the figure is available in the online version of the paper.

was further assessed using support vector machine (SVM) classification and leave-one-out cross validation (LOOCV). The four tested classifiers included as input parameters: 1) ADAS-cog score, 2) amyloid PET regional SUVRs, 3) FDG regional SUVRs, and 4) ADAS-cog score, amyloid, and FDG regional SUVRs. ADAS-cog score and PET SUVRs were processed as continuous variables. In LOOCV, each subject was classified based on an SVM model built using as a training set all subjects in groups 2 and 3 except that one.

Statistical analyses were performed using Matlab R2013 (The Math Works, Natick, MA).

\section{RESULTS}

Figure 1 shows the voxel-wise correlation map between amyloid load (florbetapir SUVR) and glucose metabolism (FDG SUVR). On each canonical view (axial, coronal, sagittal), the top row shows the correlation map with cortical ROIs superimposed, and the bottom row shows the correlation map overlaid on a T1-MR scan. Only negative correlations between amyloid and metabolic SUVRs are shown. Table 2 gives the correlation coefficient between regional amyloid and metabolic SUVRs for the eight
Table 2

Correlation between cortical amyloid SUVR and metabolic SUVR

\begin{tabular}{lcc}
\hline & \multicolumn{2}{c}{ Amyloid/metabolism correlation } \\
\cline { 2 - 3 } & Pearson's r & $p$ \\
\hline Frontal & -0.01 & $\mathrm{~ns}$ \\
Temporal & -0.21 & $<0.001$ \\
Parietal & -0.05 & $\mathrm{~ns}$ \\
Occipital & -0.02 & $\mathrm{~ns}$ \\
Insula & 0.06 & $\mathrm{~ns}$ \\
Precuneus & -0.24 & $<0.001$ \\
Ant. cingulum & 0.03 & $\mathrm{~ns}$ \\
Post. cingulum & -0.26 & $<0.001$ \\
\hline
\end{tabular}

cortical ROIs. A significant negative correlation was found in the temporal cortex $(r=-0.21 ; p<0.001)$, precuneus $(r=-0.24 ; p<0.001)$, and posterior cingulum $(r=-0.26 ; p<0.001)$.

Figure 2 shows the joint distributions (median and inter-quartile range) of amyloid load and glucose metabolism according to clinical profile in the eight cortical ROIs. Indicated F-scores are measures of the distance between normal and stable MCI, stable MCI and AD converters, and AD converters and AD, respectively. Table 3 details the relative variation (relative $\Delta_{\text {mean SUVR }}$ ) in mean regional amyloid load and glucose metabolism between (from left to right) normal and stable MCI, stable MCI and AD converters, and $\mathrm{AD}$ converters and $\mathrm{AD}$. 
A

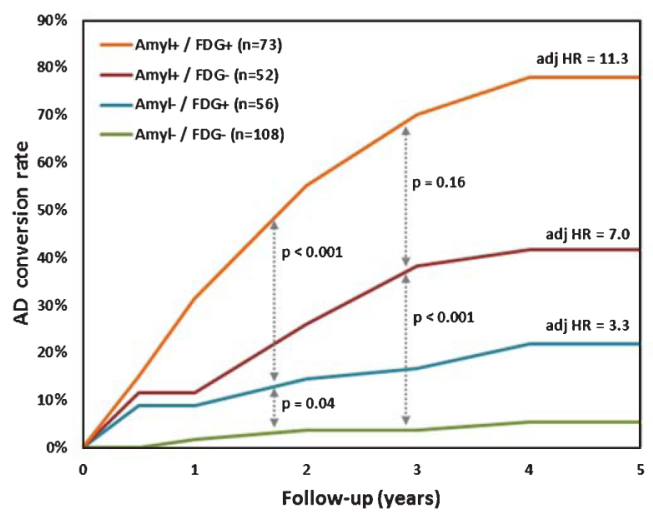

B

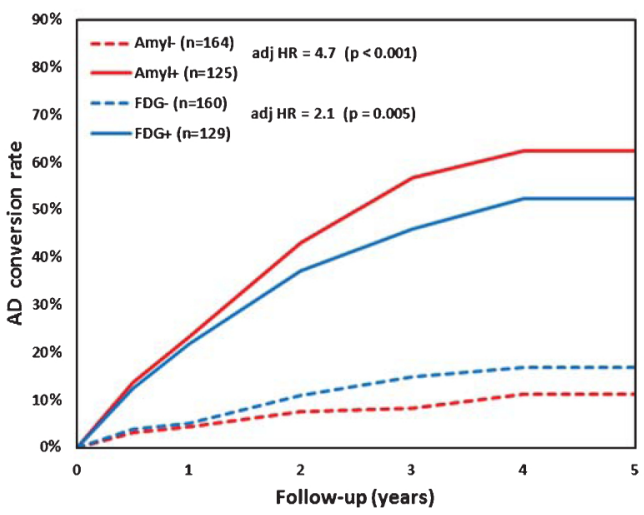

Fig. 3. Kaplan-Meier curves for conversion to AD in cognitively impaired subjects according to joint amyloid/FDG profile (A) and individual amyloid or FDG profile (B). adj HR, adjusted hazard ratio with respect to amyloid-/FDG- (A) and amyloid- or FDG- (B). Cox model accounts for age, gender, ApoE status, and baseline ADAS-cog score.

Table 4

Prognostic value of ADAS-cog score, amyloid PET regional SUVRs, FDG PET regional SUVRs, and their combination regarding conversion to $\mathrm{AD}$ in subjects with cognitive impairment

\begin{tabular}{lcccc}
\hline & ADAS-cog & $\begin{array}{c}\text { Amyloid PET } \\
\text { (8 regional } \\
\text { SUVRs) }\end{array}$ & $\begin{array}{c}\text { FDG PET } \\
\text { (8 regional } \\
\text { SUVRs) }\end{array}$ & \\
\hline Sensitivity & $71 \%$ & $76 \%$ & $71 \%$ & $74 \%$ \\
Specificity & $73 \%$ & $71 \%$ & $66 \%$ & $78 \%$ \\
Accuracy & $72 \%$ & $72 \%$ & $67 \%$ & $77 \%$ \\
PPV & $52 \%$ & $52 \%$ & $47 \%$ & $58 \%$ \\
NPV & $86 \%$ & $87 \%$ & $84 \%$ & $88 \%$ \\
\hline
\end{tabular}

PPV, positive predictive value; NPV, negative predictive value.

It yielded similar negative predictive value and sensitivity compared to ADAS-cog score and amyloid PET.

\section{DISCUSSION}

In this study based on prospective data from the ADNI-2 cohort, we systematically investigated the correlation between cortical amyloid deposition and glucose metabolism across cross-sectionally modelled disease stages. Amyloid load was assessed using florbetapir, which is one of the tracers available to this end. Although fluorinated amyloid tracers share intimately close properties in terms of binding mechanisms and pharmacokinetics, the findings reported here using florbetapir need to be verified with other fluorinated tracers. The recent literature regarding the association between amyloid load and glucose metabolism mostly focused on specific populations, yielding partial and heterogeneous infor- mation regarding the underlying pathophysiological processes. Bozoki et al. found that the effect of amyloid positivity on brain metabolism was regionally specific in healthy subjects, and that amyloid load and hypometabolism were moderately correlated in the temporal cortex in ApoE4 carriers [19]. In a small cohort of healthy and AD subjects, La Joie et al. found no significant correlation between amyloid load and hypometabolism [17]. In AD subjects, regional profiles were characterized by predominant amyloid load with high hypometabolism in posterior association areas, and maximal amyloid deposition with mild hypometabolism in frontal regions. Other studies focused specifically on MCI subjects. In the study by Wu et al. [14], early MCI subject showed increased amyloid load in frontal, precuneus, and posterior cingulate cortices compared to healthy controls. Late MCI subjects showed decreased metabolism in the precuneus and temporo-parietal cortices compared to early MCI. In a cohort of MCI patients with family history of AD, Mosconi et al. found that amyloid load was the most pronounced anomaly in most of cortical areas, except for medial temporal and posterior cingulate regions where hypometabolism was predominant [18]. In a small sample of healthy, MCI, and AD subjects, Jagust et al. found a moderate $62 \%$ agreement between amyloid and FDG profiles based on a dichotomous global assessment of cortical tracer uptake, but did not examine in detail regional fluctuations and their inter-correlation [22].

The results presented in the previous section tend to substantiate these findings. Both at the voxel and regional scales, the correlation between amyloid load and hypometabolism was prominent in temporal, 
precuneus and posterior cingulate cortices, and not significant in fronto-parietal and occipital areas. A moderate positive correlation between amyloid and FDG SUVRs was observed in superficial and periventricular regions, and was considered as an artefact related to partial volume effect (PVE)-related uptake decrease in subjects with cortical and subcortical atrophy. These observations tend to attest that amyloid load increase and glucose metabolism decrease evolve concomitantly in some cortical areas (temporal cortex, precuneus and posterior cingulum), at least over a given period of AD natural history [33]. As reported in Table 3, these three cortical areas are those where a highly significant change in both amyloid and FDG SUVR was found between stable MCI and $\mathrm{AD}$ converters, consistent with prior studies [14, 18]. At the prodromal stage, significant albeit subtle modifications in joint amyloid/FDG profile occur, particularly in the precuneus cortex $(+7 \%$ in amyloid SUVR and $-3 \%$ in FDG SUVR in stable MCI versus normal subjects). At the symptomatic stage, amyloid load increase was diffuse and early (about $+20 \%$ from stable $\mathrm{MCI}$ to $\mathrm{AD}$ converters; no significant change between $\mathrm{AD}$ converters and $\mathrm{AD}$ ), whereas metabolism decrease proceeded later and predominated in temporo-parietal, precuneus and posterior cingulate cortices (sustained significant changes in these areas from stable MCI to AD). Regional FDG hypometabolism reflects synaptic dysfunction, which may be induced by both the local toxic effects of amyloid pathology and tangle-related neurodegeneration. As already suggested in a recent study using a large cohort with cross-sectionally modelled disease stages, it seems that hypometabolism initiate within regions of highest amyloid deposition and spreads among regions most affected by amyloid pathology [34].

Some recent research has highlighted the potential diagnostic value of combined amyloid and FDG imaging. Using multimodal principal component analysis, Laforce et al. showed that joint assessment of amyloid and FDG patterns yielded higher accuracy in subject classification than unimodal assessment of amyloid or FDG profile [35]. Takahashi et al. investigated the usefulness of metabolism to amyloid SUV ratio [36]. Using cortical ROI analysis to classify healthy and AD subjects, they found that the SUV ratio allowed higher diagnostic accuracy $(83 \%)$ than FDG SUV (81\%), and amyloid SUV (79\%) individually.

Here, we specifically focused on the prognostic value of joint amyloid and FDG PET profile in terms of $\mathrm{AD}$ conversion in cognitively impaired patients. Interestingly, the five-year conversion rates were approximately in the ratio $1 / 2 / 4$ in amyloid-/FDG+, amyloid+/FDG-, and amyloid+/FDG+ subjects $(22 \%, 42 \%$, and $78 \%$ respectively), consistent with those reported by Caroli et al. [37]. Within a given FDG profile, the positivity of amyloid profile had a significant impact on the conversion adjusted hazard ratio $(p<0.001$ in both FDG- and FDG+ subjects), attesting that amyloid PET is a strong independent predictor of cognitive decline, especially at early stages of the disease.

In amyloid positive subjects, the positivity of FDG profile marginally influenced the adjusted hazard ratio of $\mathrm{AD}$ conversion $(p=0.16)$. This relatively low impact of FDG profile was likely due to the adjustment on cognitive status (through ADAS-cog score), since FDG pattern anomalies are usually correlated with significant and measurable cognitive impairment. Nonetheless, our results confirm that hypometabolism indicates a more advanced stage of the disease: in subjects with documented amyloidopathy, the five-year AD conversion rate is almost two-fold higher when glucose metabolism alterations are evidenced.

On the contrary, in amyloid negative subjects, the positivity of FDG profile significantly impacted the adjusted hazard ratio $(p=0.04)$. Among the 164 subjects with a negative amyloid profile, $16(10 \%)$ evolved to a demented state during follow-up, 11 $(69 \%)$ of whom had an abnormal FDG profile. These atypical profiles were likely related to AD-like dementia without underlying amyloidopathy, often referred to as tangle-predominant dementia [11]. Recent studies highlighted that a substantial proportion of subjects with a primary clinical diagnosis of mild to moderate $\mathrm{AD}$ have minimal $\mathrm{A} \beta$ plaque accumulation, almost a half of whom have an extensive topographic distribution of neurofibrillary degeneration at autopsy [38]. This is consistent with our finding that FDG profile allows to rectify about two thirds of amyloid PET false negatives. Regarding the predictive value of the available markers, we showed that combining clinical cognitive assessment (which is known to be a major prognostic factor [39]) with information from quantitative amyloid PET and quantitative FDG PET yields higher accuracy in predicting $\mathrm{AD}$ conversion (almost $80 \%$ ) than FDG PET, amyloid PET, and ADAS-cog score individually (about $70 \%$ accuracy for these three markers). While maintaining an excellent negative predictive value 
$(\sim 90 \%)$, it allowed a substantial increase in positive predictive value (almost $60 \%$ versus $45-50 \%$ for individual markers). These findings support the previous evidence that combining biomarkers allows better prediction of cognitive decline in subjects at risk and improves MCI subject classification with respect to $\mathrm{AD}$ conversion [23, 24, 40]. To allow for efficient discrimination between neurodegenerative diseases and optimal therapeutic planning, joint assessment of FDG and amyloid PET has to be integrated within the range of available biomarkers, including tau-specific PET tracers currently under clinical assessment [41].

\section{CONCLUSION}

The characterization of spatial distribution and temporal progression of amyloid deposits and neuronal hypometabolism is essential for elucidating pathological mechanisms that underlie AD. This study based on the analysis of cross-sectionally modelled disease stages tends to support the early and diffuse amyloid accumulation and later glucose metabolism alteration in AD natural history. It suggests that, at least over a given time range in the evolution from the prodromal state to the demented state, amyloidopathy and hypometabolism evolve concomitantly in specific cortical areas (temporal cortex, precuneus, and posterior cingulate). In a prognostic perspective, amyloid PET profile appears as the main predictor of subsequent cognitive decline and $\mathrm{AD}$ conversion in cognitively impaired subjects. In subjects with a normal amyloid profile, FDG profile allows to detect about two thirds of amyloid PET false negatives. When forecasting AD conversion using binary linear classification, the optimal predictive accuracy is obtained by combining baseline cognitive status, regional amyloid SUVR, and regional FDG SUVR.

\section{ACKNOWLEDGMENTS}

Data collection and sharing for this project was funded by the Alzheimer's Disease Neuroimaging Initiative (ADNI) (National Institutes of Health Grant U01 AG024904) and DOD ADNI (Department of Defense award number W81XWH-12-2-0012). ADNI is funded by the National Institute on Aging, the National Institute of Biomedical Imaging and Bioengineering, and through generous contributions from the following: AbbVie, Alzheimer's Association; Alzheimer's Drug Discovery Foundation;
Araclon Biotech; BioClinica, Inc.; Biogen; BristolMyers Squibb Company; CereSpir, Inc.; Cogstate; Eisai Inc.; Elan Pharmaceuticals, Inc.; Eli Lilly and Company; EuroImmun; F. Hoffmann-La Roche Ltd and its affiliated company Genentech, Inc.; Fujirebio; GE Healthcare; IXICO Ltd.; Janssen Alzheimer Immunotherapy Research \& Development, LLC.; Johnson \& Johnson Pharmaceutical Research \& Development LLC.; Lumosity; Lundbeck; Merck \& Co., Inc.; Meso Scale Diagnostics, LLC.; NeuroRx Research; Neurotrack Technologies; Novartis Pharmaceuticals Corporation; Pfizer Inc.; Piramal Imaging; Servier; Takeda Pharmaceutical Company; and Transition Therapeutics. The Canadian Institutes of Health Research is providing funds to support ADNI clinical sites in Canada. Private sector contributions are facilitated by the Foundation for the National Institutes of Health (http://www.fnih.org). The grantee organization is the Northern California Institute for Research and Education, and the study is coordinated by the Alzheimer's Therapeutic Research Institute at the University of Southern California. ADNI data are disseminated by the Laboratory for Neuro Imaging at the University of Southern California.

\section{REFERENCES}

[1] Small SA, Duff K (2008) Linking Abeta and tau in late-onset Alzheimer's disease: A dual pathway hypothesis. Neuron 60, 534-542.

[2] Karran E, Mercken M, De Strooper B (2011) The amyloid cascade hypothesis for Alzheimer's disease: An appraisal for the development of therapeutics. Nat Rev Drug Discov 10, 698-712.

[3] Petersen RC, Aisen P, Boeve BF, Geda YE, Ivnik RJ, Knopman DS, Mielke M, Pankratz VS, Roberts R, Rocca WA, Weigand S, Weiner M, Wiste H, Jack CR Jr (2013) Mild cognitive impairment due to Alzheimer disease in the community. Ann Neurol 74, 199-208.

[4] Ellendt S, Voß B, Kohn N, Wagels L, Goerlich K, Drexler E, Schneider F, Habel U (2017) Predicting stability of Mild Cognitive Impairment (MCI): Findings of a community based sample. Curr Alzheimer Res 14, 608-619.

[5] Frisoni GB, Bocchetta M, Chételat G, Rabinovici GD, de Leon MJ, Kaye J, Reiman EM, Scheltens P, Barkhof F, Black SE, Brooks DJ, Carrillo MC, Fox NC, Herholz K, Nordberg A, Jack CR, Jagust WJ, Johnson KA, Rowe CC, Sperling RA, Thies W, Wahlund LO, Weiner MW, Pasqualetti P, Decarli C; ISTAART's NeuroImaging Professional Interest Area (2013) Imaging markers for Alzheimer disease: Which vs how. Neurology 81, 487-500.

[6] Boccardi M, Bocchetta M, Apostolova LG, Barnes J, Bartzokis G, Corbetta G, DeCarli C, deToledo-Morrell L, Firbank M, Ganzola R, Gerritsen L, Henneman W, Kil- 
liany RJ, Malykhin N, Pasqualetti P, Pruessner JC, Redolfi A, Robitaille N, Soininen H, Tolomeo D, Wang L, Watson C, Wolf H, Duvernoy H, Duchesne S, Jack CR Jr, Frisoni GB (2015) Delphi definition of the EADC-ADNI Harmonized Protocol for hippocampal segmentation on magnetic resonance. Alzheimers Dement 11, 126-138.

[7] Clark CM, Pontecorvo MJ, Beach TG, Bedell BJ, Coleman RE, Doraiswamy PM, Fleisher AS, Reiman EM, Sabbagh MN, Sadowsky CH, Schneider JA, Arora A, Carpenter AP, Flitter ML, Joshi AD, Krautkramer MJ, Lu M, Mintun MA, Skovronsky DM (2012) Cerebral PET with florbetapir compared with neuropathology at autopsy for detection of neuritic amyloid- $\beta$ plaques: A prospective cohort study. Lancet Neurol 11, 669-678.

[8] Minoshima S, Drzezga AE, Barthel H, Bohnen N, Djekidel M, Lewis DH, Mathis CA, McConathy J, Nordberg A, Sabri O, Seibyl JP, Stokes MK, Van Laere K (2016) SNMMI Procedure Standard/EANM Practice Guideline for Amyloid PET Imaging of the Brain 1.0. J Nucl Med 57, 1316-1322.

[9] Silverman DH, Small GW, Chang CY, Lu CS, Kung De Aburto MA, Chen W, Czernin J, Rapoport SI, Pietrini P, Alexander GE, Schapiro MB, Jagust WJ, Hoffman JM, Welsh-Bohmer KA, Alavi A, Clark CM, Salmon E, de Leon MJ, Mielke R, Cummings JL, Kowell AP, Gambhir SS, Hoh CK, Phelps ME (2001) Positron emission tomography in evaluation of dementia: Regional brain metabolism and long-term outcome. JAMA 286, 2120-2127.

[10] Perani D, Della Rosa PA, Cerami C, Gallivanone F, Fallanca F, Vanoli EG, Panzacchi A, Nobili F, Pappatá S, Marcone A, Garibotto V, Castiglioni I, Magnani G, Cappa SF, Gianolli L (2014) Validation of an optimized SPM procedure for FDGPET in dementia diagnosis in a clinical setting. Neuroimage Clin 6, 445-454.

[11] Morris GP, Clark IA, Vissel B (2014) Inconsistencies and controversies surrounding the amyloid hypothesis of Alzheimer's disease. Acta Neuropathol Commun 2, 135.

[12] Jack CR Jr, Knopman DS, Jagust WJ, Shaw LM, Aisen PS, Weiner MW, Petersen RC, Trojanowski JQ (2010) Hypothetical model of dynamic biomarkers of the Alzheimer's pathological cascade. Lancet Neurol 9, 119-128.

[13] Förster S, Grimmer T, Miederer I, Henriksen G, Yousefi BH, Graner P, Wester HJ, Förstl H, Kurz A, Dickerson BC, Bartenstein P, Drzezga A (2012) Regional expansion of hypometabolism in Alzheimer's disease follows amyloid deposition with temporal delay. Biol Psychiatry 71, 792-797.

[14] Wu L, Rowley J, Mohades S, Leuzy A, Dauar MT, Shin M, Fonov V, Jia J, Gauthier S, Rosa-Neto P (2012) Dissociation between brain amyloid deposition and metabolism in early mild cognitive impairment. PLoS One 7, e47905.

[15] Kadir A, Almkvist O, Forsberg A, Wall A, Engler H, Långström B, Nordberg A (2012) Dynamic changes in PET amyloid and FDG imaging at different stages of Alzheimer's disease. Neurobiol Aging 33, 198.e1-14.

[16] Ossenkoppele R, Tolboom N, Foster-Dingley JC, Adriaanse SF, Boellaard R, Yaqub M, Windhorst AD, Barkhof F, Lammertsma AA, Scheltens P, van der Flier WM, van Berckel BN (2013) Longitudinal imaging of Alzheimer pathology using [11C]PIB, [18F]FDDNP and [18F]FDG PET. Eur J Nucl Med Mol Imaging 39, 990-1000.

[17] La Joie R, Perrotin A, Barré L, Hommet C, Mézenge F, Ibazizene M, Camus V, Abbas A, Landeau B, Guilloteau D, de La Sayette V, Eustache F, Desgranges B, Chételat G (2012) Region-specific hierarchy between atrophy, hypometabolism, and $\beta$-amyloid $(A \beta)$ load in
Alzheimer's disease dementia. JNeurosci 32, 16265-16273.

[18] Mosconi L, Andrews RD, Matthews DC (2013) Comparing brain amyloid deposition, glucose metabolism, and atrophy in mild cognitive impairment with and without a family history of dementia. J Alzheimers Dis 35, 509-524.

[19] Bozoki AC, Zdanukiewicz M, Zhu DC, Alzheimer's Disease Neuroimaging Initiative (2016) The effect of $\beta$-amyloid positivity on cerebral metabolism in cognitively normal seniors. Alzheimers Dement 12, 1250-1258.

[20] Edison P, Archer HA, Hinz R, Hammers A, Pavese N, Tai YF, Hotton G, Cutler D, Fox N, Kennedy A, Rossor M, Brooks DJ (2007) Amyloid, hypometabolism, and cognition in Alzheimer disease: An [11C]PIB and [18F]FDG PET study. Neurology 68, 501-508.

[21] Shin J, Lee SY, Kim SJ, Kim SH, Cho SJ, Kim YB (2010) Voxel-based analysis of Alzheimer's disease PET imaging using a triplet of radiotracers: PIB, FDDNP, and FDG. Neuroimage 52, 488-496.

[22] Jagust WJ, Landau SM, Shaw LM, Trojanowski JQ, Koeppe RA, Reiman EM, Foster NL, Petersen RC, Weiner MW, Price JC, Mathis CA (2009) Relationships between biomarkers in aging and dementia. Neurology 73, 11931199.

[23] Iaccarino L, Chiotis K, Alongi P, Almkvist O, Wall A, Cerami C, Bettinardi V, Gianolli L, Nordberg A, Perani D (2017) A cross-validation of FDG- and amyloid-PET biomarkers in mild cognitive impairment for the risk prediction to dementia due to Alzheimer's disease in a clinical setting. J Alzheimers Dis 59, 603-614.

[24] Prestia A, Caroli A, Wade SK, van der Flier WM, Ossenkoppele R, Van Berckel B, Barkhof F, Teunissen CE, Wall A, Carter SF, Schöll M, Choo IH, Nordberg A, Scheltens P, Frisoni GB (2015) Prediction of AD dementia by biomarkers following the NIA-AA and IWG diagnostic criteria in MCI patients from three European memory clinics. Alzheimers Dement 11, 1191-1201.

[25] Landau SM, Mintun MA, Joshi AD, Koeppe RA, Petersen RC, Aisen PS, Weiner MW, Jagust WJ (2012) Amyloid deposition, hypometabolism, and longitudinal cognitive decline. Ann Neurol 72, 578-586.

[26] Petersen RC, Aisen PS, Beckett LA, Donohue MC, Gamst AC, Harvey DJ, Jack CR Jr, Jagust WJ, Shaw LM, Toga AW, Trojanowski JQ, Weiner MW (2010) Alzheimer's Disease Neuroimaging Initiative (ADNI): Clinical characterization. Neurology 74, 201-209.

[27] McKhann G, Drachman DA, Folstein M, Katzman R, Price DL, Stadlan EM (1984) Clinical diagnosis of Alzheimer's disease-report of the NINCDS-ADRDA work group under the auspices of Department of Health and Human Services Task Force on Alzheimer's disease. Neurology 34, 939-944.

[28] Dubois B, Feldman HH, Jacova C, DeKosky ST, BarbergerGateau P, Cummings J, Delacourte A, Galasko D, Gauthier S, Jicha G, Meguro K, O'brien J, Pasquier F, Robert P, Rossor M, Salloway S, Stern Y, Visser PJ, Scheltens P (2007) Research criteria for the diagnosis of Alzheimer's disease: Revising the NINCDS-ADRDA criteria. Lancet Neurol 6, 734-746.

[29] Landau SM, Breault C, Joshi AD, Pontecorvo M, Mathis CA, Jagust WJ, Mintun MA (2013) Amyloid-beta imaging with Pittsburgh compound B and florbetapir: Comparing radiotracers and quantification methods. J Nucl Med 54, 70-77.

[30] Dukart J, Mueller K, Horstmann A, Vogt B, Frisch S, Barthel H, Becker G, Möller HE, Villringer A, Sabri O, Schroeter 
ML (2010) Differential effects of global and cerebellar normalization on detection and differentiation of dementia in FDG-PET studies. Neuroimage 49, 1490-1495.

[31] Bullich S, Villemagne VL, Catafau AM, Jovalekic A, Koglin N, Rowe CC, De Santi S (2017) Optimal reference region to measure longitudinal amyloid-beta change with $18 \mathrm{~F}-$ florbetaben PET. J Nucl Med 58, 1300-1306.

[32] Marcus C, Mena E, Subramaniam RM (2014) Brain PET in the diagnosis of Alzheimer's disease. Clin Nucl Med 39, e413-e422.

[33] Förster S, Grimmer T, Miederer I, Henriksen G, Yousefi BH, Graner P, Wester HJ, Förstl H, Kurz A, Dickerson BC, Bartenstein P, Drzezga A (2012) Regional expansion of hypometabolism in Alzheimer's disease follows amyloid deposition with temporal delay. Biol Psychiatry 71, 792-797.

[34] Grothe MJ, Teipel SJ, Alzheimer's Disease Neuroimaging Initiative (2016) Spatial patterns of atrophy, hypometabolism, and amyloid deposition in Alzheimer's disease correspond to dissociable functional brain networks. Hum Brain Mapp 37, 35-53.

[35] Laforce R Jr, Tosun D, Ghosh P, Lehmann M, Madison CM, Weiner MW, Miller BL, Jagust WJ, Rabinovici GD (2014) Parallel ICA of FDG-PET and PiB-PET in three conditions with underlying Alzheimer's pathology. Neuroimage Clin 4, 508-516.

[36] Takahashi R, Ishii K, Yokoyama K, Alzheimer's Disease Neuroimaging Initiative (2017) Validation of a new imaging technique using the glucose metabolism to amyloid deposition ratio in the diagnosis of Alzheimer's disease. Curr Alzheimer Res 14, 161-168.
[37] Caroli A, Prestia A, Galluzzi S, Ferrari C, van der Flier WM, Ossenkoppele R, Van Berckel B, Barkhof F, Teunissen C, Wall AE, Carter SF, Schöll M, Choo IH, Grimmer T, Redolfi A, Nordberg A, Scheltens P, Drzezga A, Frisoni GB (2015) Mild cognitive impairment with suspected nonamyloid pathology (SNAP): Prediction of progression. Neurology 84, 508-515.

[38] Monsell SE, Kukull WA, Roher AE, Maarouf CL, Serrano G, Beach TG, Caselli RJ, Montine TJ, Reiman EM (2015) Characterizing apolipoprotein $\mathrm{E} \varepsilon 4$ carriers and noncarriers with the clinical diagnosis of mild to moderate Alzheimer dementia and minimal $\beta$-amyloid peptide plaques. JAMA Neurol 72, 1124-1131.

[39] Ben Bouallègue F, Mariano-Goulart $D$, Payoux $P$, Alzheimer's Disease Neuroimaging Initiative (2017) Comparison of CSF markers and semi-quantitative amyloid PET in Alzheimer's disease diagnosis and in cognitive impairment prognosis using the ADNI-2 database. Alzheimers Res Ther 9, 32 .

[40] Shaffer JL, Petrella JR, Sheldon FC, Choudhury KR, Calhoun VD, Coleman RE, Doraiswamy PM (2013) Predicting cognitive decline in subjects at risk for Alzheimer disease by using combined cerebrospinal fluid, MR imaging, and PET biomarkers. Radiology 266, 583-591.

[41] Saint-Aubert L, Lemoine L, Chiotis K, Leuzy A, RodriguezVieitez E, Nordberg A (2017) Tau PET imaging: Present and future directions. Mol Neurodegener 12, 19. 